\title{
L'alphabétisation des adultes : le cadre international
}

\section{Héloïse Nétange}

\section{OpenEdition}

1 Journals

Édition électronique

URL : https://journals.openedition.org/ries/2042

DOI : $10.4000 /$ ries.2042

ISSN : 2261-4265

Éditeur

France Education international

\section{Édition imprimée}

Date de publication : 1 septembre 2011

Pagination : 12-15

ISBN : 978-2-85420-592-3

ISSN : $1254-4590$

\section{Référence électronique}

Héloïse Nétange, «L'alphabétisation des adultes : le cadre international », Revue internationale d'éducation de Sèvres [En ligne], 57 | septembre 2011, mis en ligne le 01 septembre 2011, consulté le 05 juillet 2021. URL : http://journals.openedition.org/ries/2042; DOI : https://doi.org/10.4000/ries. 2042

Ce document a été généré automatiquement le 5 juillet 2021.

(c) Tous droits réservés 


\title{
L'alphabétisation des adultes : le cadre international
}

\author{
Héloïse Nétange
}

1 D'après l'Unesco, 759 millions de jeunes et d'adultes sont aujourd'hui dépourvus de compétences d'alphabétisation. Selon la définition de l'Unesco de 1958, est analphabète «toute personne incapable de lire et d'écrire, en le comprenant, un exposé bref et simple de faits en rapport avec la vie quotidienne». D'autres approches de l'analphabétisme ont été pensées par la suite, intégrant les notions de compétences et de leurs applications pratiques. Le terme «illettrisme » est également employé pour désigner généralement le fait d'avoir été scolarisé sans avoir pour autant acquis les compétences de base (lire, écrire, compter). La définition de ces notions continue à s'enrichir, et plusieurs courants de pensée coexistent.

2 Les organisations internationales ont tenté, avec des approches différentes, de sensibiliser et d'inciter à la lutte contre l'analphabétisme. Cet article est le premier volet d'une sélection de ressources en ligne sur la question de l'alphabétisation des adultes. Il propose un aperçu du cadre et des programmes en cours menés par quelques grands organismes internationaux: l'UNESCO, la Commission européenne et l'OCDE. Dans le prochain numéro, nous donnerons des exemples de politiques publiques mises en place dans des pays du Nord et du Sud.

3 Sitographie arrêtée le 18 juillet 2011.

\section{UNESCO}

4 L'alphabétisation, un enjeu vital, Rapport mondial de suivi sur l'Éducation pour tous, 2006

5 Chaque année depuis 2002, l'UNESCO publie un Rapport mondial de suivi de l'éducation pour tous (EPT), examinant les progrès réalisés en direction des six objectifs de l'EPT adoptés en 2000 au Forum mondial sur l'éducation de Dakar. Le rapport 2006 s'est penché sur l'alphabétisation. Soulignant qu'il s'agit d'un droit d'une importance 
cruciale pour le développement, il appelle à une intensification des programmes d'alphabétisation. Par son approche historique, sa description des différentes approches de la notion d'analphabétisme et ses données chiffrées, c'est un ouvrage de synthèse fondamental sur l'état de l'alphabétisation dans le monde. Il est téléchargeable en ligne: http://www.unesco.org/new/fr/education/themes/leadingthe-international-agenda/efareport/reports/2006-literacy/

\section{La Décennie des Nations Unies pour l'alphabétisation (DNUA) 2003-2012}

6 L'UNESCO est le coordonnateur international de la DNUA dont le thème est «L'alphabétisation, source de liberté ». L'objectif général de la DNUA est celui fixé par l'Objectif 4 de l'EPT : accroître de $50 \%$ le taux d'alphabétisation d'ici à 2015. Elle a adopté cinq thèmes biennaux liés à l'alphabétisation: le genre, le développement durable, la santé, l'employabilité et la paix: http://www.unesco.org/new/fr/ education/themes/education-building-blocks/literacy/un-literacy-decade/

\section{Institut de l'UNESCO pour l'apprentissage tout au long de la vie} (UIL)

7 L'Institut de l'UNESCO pour l'apprentissage tout au long de la vie (UIL) est l'un des six centres de l'UNESCO spécialisés dans l'éducation. Il concentre son action sur l'éducation des adultes, en particulier l'alphabétisation et l'éducation non formelle ainsi que les possibilités d'un apprentissage alternatif pour les groupes marginalisés et défavorisés. Bien que l'UIL travaille essentiellement avec l'Afrique et les pays en voie de développement, il a été l'une des premières organisations à examiner l'alphabétisme fonctionnel des adultes en Europe. Il coordonne plusieurs actions que nous détaillons ci-dessous. C'est également le centre de ressources de l'UNESCO en matière d'alphabétisation et d'éducation non formelle. De nombreuses publications sont téléchargeables en ligne: http://uil.unesco.org/fr/accueil/domaines-dactivites/ alphabetisation/

\section{Initiative pour l'alphabétisation : savoir pour pouvoir (LIFE) 2006-2015}

8 L'Initiative de l'UNESCO pour l'alphabétisation: savoir pour pouvoir (LIFE) sert de cadre à la mise en œuvre de la DNUA. Elle accorde une attention particulière à l'alphabétisme des adultes et des enfants non scolarisés et cible les trente cinq pays dans lesquels le taux d'alphabétisation est inférieur à $50 \%$ ou qui comptent plus de dix millions de personnes ne sachant ni lire ni écrire. $85 \%$ de la population analphabète mondiale, dont deux tiers de femmes et de filles, vit dans ces pays. Un bilan sur les trois premières années d'action du programme (2006-2009) a permis d'évaluer les premiers résultats de l'amélioration des conditions de vie des apprenants à travers le monde : http://uil.unesco.org/fr/accueil/domaines-dactivites/alphabetisation/life-initiativepour-lalphabetisation-savoir-pour-pouvoir/ 


\section{Litbase - Pratiques efficaces d'alphabétisation}

Litbase est une plateforme en ligne proposant une sélection de programmes efficaces en matière d'alphabétisation et de numératie des adultes, issus de toutes les régions du monde. Elle a pour vocation l'échange d'expériences et le transfert de connaissances. On peut y faire une recherche par pays, thèmes et groupes-cibles. Chaque projet y est décrit de manière précise: profil du pays avec des chiffres clés, présentation du contexte du programme, de ses objectifs, des différentes phases de réalisation du programme, de son suivi, de son évaluation et des résultats observés. Enfin, les éléments novateurs et les leçons apprises sont mis en exergue: http:// www.unesco.org/uil/litbase/

\section{Programme de suivi et d'évaluation de l'alphabétisation (LAMP)}

10 En 2003, l'Institut de statistique de l'UNESCO (ISU) a entrepris l'élaboration du Programme d'évaluation et de suivi de l'alphabétisation (LAMP), afin de recueillir des données fiables sur l'alphabétisation dans les pays en développement au moyen d'enquêtes menées à l'échelle nationale auprès des ménages. Les méthodes de mesure des compétences élaborées doivent permettre des comparaisons, malgré la différence de contextes linguistiques et de développement : http://www.uis.unesco.org/Literacy/ Pages/lamp-literacy-assessment.aspx

\section{Conférence internationale sur l'éducation des adultes (CONFINTEA VI)}

11 Depuis la fin des années 1940, l'UNESCO organise tous les douze ou treize ans des réunions internationales sur l'éducation des adultes. La Sixième Conférence internationale sur l'éducation des adultes (CONFINTEA VI) s'est tenue au Brésil en décembre 2009. C'est un espace de dialogue en matière de politiques de promotion de l'apprentissage et de l'éducation non formelle des adultes, dont l'alphabétisation est la base. Les actes de cette conférence, ainsi que le Rapport mondial sur l'apprentissage et l'éducation des adultes, synthétisant 154 rapports nationaux sont téléchargeables en ligne : http://www.unesco.org/fr/confinteavi/

\section{Commission européenne}

Bien qu'aujourd'hui une grande majorité des Européens aient accès à l'école, l'illettrisme est encore répandu dans les pays de l'Union européenne. En effet, 80 millions de personnes auraient du mal à lire et à écrire. L'Union européenne intervient dans le domaine de l'alphabétisation à un niveau politique, en définissant des orientations générales et des objectifs pour la formation des adultes et l'éducation, ainsi qu'en soutenant des projets. 


\section{Recommandation du Parlement européen et du Conseil, sur les compétences clés pour l'éducation et la formation tout au long de la vie}

13 Cette recommandation du 18 décembre 2006 définit huit compétences clés « dont chaque personne a besoin pour obtenir un emploi et parvenir à la satisfaction personnelle, à l'inclusion sociale et à la citoyenneté active dans le monde actuel axé sur la connaissance ». Les projets de l'Union européenne en vue de relever le niveau d'alphabétisation sont liés aux initiatives phares de la stratégie Europe 2020 "Jeunesse en mouvement » et "Une stratégie pour des compétences nouvelles et des emplois », visant toutes deux à améliorer le niveau de qualification en Europe en améliorant la qualité de l'éducation et de la formation: http://ec.europa.eu/education/lifelonglearning-policy/doc1120_fr.htm

\section{Nouvelle campagne de lutte contre l'illettrisme}

En juillet 2010, un Avis du Comité des régions sur le thème « Faire reculer l'illettrisme Penser une stratégie européenne" a émis des préconisations pour accentuer l'inscription de la lutte contre l'illettrisme dans les orientations stratégiques communautaires. En février 2011, la Commission européenne a lancé une nouvelle campagne de lutte contre l'illettrisme et mis en place un groupe d'experts indépendants chargé de "conférer visibilité et importance politique au redressement du niveau d'alphabétisation en Europe ». Ce dernier se réunira régulièrement et soumettra ses propositions à la Commission dans le courant de l'année 2012 : http:// europa.eu/legislation_summaries/education_training_youth/lifelong_learning/ c11090_fr.htm

\section{Eur-Alpha, un exemple de projet soutenu par la Commission européenne}

15 La Commission européenne finance des actions d'alphabétisation, notamment dans le cadre des programmes Grundtvig et Leonardo da Vinci. Eur-Alpha est un exemple de projet soutenu dans le cadre du programme Grundtvig. Il s'agit d'un réseau européen d'organismes ayant pour but d'améliorer la qualité de l'alphabétisation des adultes. Son action vise à favoriser les échanges entre les acteurs de terrain : les formateurs d'une part, les apprenants d'autre part. Fondé en 2009, il compte à ce jour seize partenaires issus de douze pays. Il organise notamment des ateliers réunissant des apprenants en alphabétisation, pour développer un réseau d'échanges entre des groupes de personnes qui sont en cours d'apprentissage des compétences de base : http://www.eur-alpha.eu/

\section{Organisation de coopération et de développement économiques (OCDE)}

16 Il y a quelques années, l'OCDE a mené plusieurs grandes enquêtes internationales sur l'illettrisme : l'enquête International Adult Literacy Survey (IALS) dans les années quatre- 
vingt-dix, et Adult Literacy and Lifeskills (ALL) menée en 2003 et 2005. La stratégie actuelle se tourne davantage vers les compétences des adultes.

\section{Stratégie de l'OCDE sur les compétences}

17 Dans sa nouvelle stratégie sur les compétences, l'OCDE souligne que, parmi ses pays membres, « l'illettrisme au sein de la population adulte reste un problème majeur dans beaucoup de pays à revenu faible ou intermédiaire ». L'accent est mis sur l'importance d'avoir une main-d'œuvre qualifiée pour assurer la productivité et le développement dans un contexte d'économie mondialisée. Cette stratégie sera mise en œuvre à partir de 2013 : www.oecd.org/education/SkillsStrategy

\section{Programme pour l'évaluation internationale des compétences des adultes (PIAAC)}

Ce nouveau programme de l'OCDE a pour ambition d'évaluer le niveau et la répartition des compétences cognitives et professionnelles des adultes dans les différents pays de l'OCDE. Il évaluera ainsi le « littérisme à l'ère de l'information », c'est-à-dire "l'intérêt, l'attitude et les capacités dont font preuve les personnes pour bien utiliser les outils socioculturels, y compris les technologies numériques et les outils de communication, afin d'accéder à l'information, de la gérer, de l'assimiler et de l'évaluer, de construire de nouveaux savoirs et de communiquer avec autrui ». Cette enquête menée en 2011, et dont les résultats seront diffusés début 2013, portera sur cinq mille personnes âgées de 16 à 65 ans dans chaque pays participant : www.oecd.org/piaac/

\section{INDEX}

Mots-clés : alphabétisation, formation des adultes

Keywords : literacy, adult education

Palabras claves : alfabetización, educación de adultos

\section{AUTEUR}

\section{HÉLOÏSE NÉTANGE}

Chargée de veille au centre de ressources et d'ingénierie documentaires du CIEP. 